\title{
Genome sequencing and phylogenetic analysis of allotetraploid Salix matsudana Koidz
}

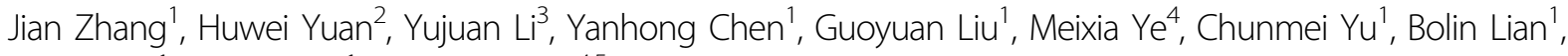
Fei Zhong ${ }^{1}$, Yuna Jiang ${ }^{1}$ and Jichen $\mathrm{Xu}^{4,5}$

\begin{abstract}
Polyploidy is a common phenomenon among willow species. In this study, genome sequencing was conducted for Salix matsudana Koidz (also named Chinese willow), an important greening and arbor tree species, and the genome of this species was compared with those of four other tree species in Salicaceae. The total genome sequence of $S$. matsudana was $655.72 \mathrm{Mb}$ in size, with repeated sequences accounting for $45.97 \%$ of the total length. In total, $531.43 \mathrm{Mb}$ of the genome sequence could be mapped onto 38 chromosomes using the published genetic map as a reference. The genome of $S$. matsudana could be divided into two groups, the A and B genomes, through homology analysis with the genome of Populus trichocarpa, and the A and B genomes contained 23,985 and 25,107 genes, respectively. 4DTv combined transposon analysis predicted that allotetraploidy in S. matsudana appeared $\sim 4$ million years ago. The results from this study will help reveal the evolutionary history of $S$. matsudana and lay a genetic basis for its breeding.
\end{abstract}

\section{Introduction}

Willows, the generic term for tree species in Salix. L and Chosenia Nakai of Salicaceae, include deciduous arbors and shrubs mainly distributed in temperate and frigid zones of the Northern Hemisphere. Willow tree species play important roles in energy production, afforestation, and greening. To date, over 520 willow tree species have been discovered worldwide. Asia is the center of origin for willow, and 257 willow tree species are distributed in China ${ }^{1,2}$. Willow tree species are resistant to a number of stresses, including salt stress $^{3}$, drought stress $^{4}$, water stress $^{5}$, and heavy metal stress ${ }^{6}$, and provide important functions in ecological restoration ${ }^{7}$.

The genetic background of willow is complex. Chromosome ploidy, including diploidy $(2 n=12 \mathrm{x}=38)$,

\footnotetext{
Correspondence: Jian Zhang (yjnkyy@163.com) or Jichen Xu (1795228030@qq. com)

${ }^{1}$ Key Lab of Landscape Plant Genetics and Breeding, School of Life Science, Nantong University, 226019 Nantong, China

${ }^{2}$ State Key Laboratory of Subtropical Silviculture, Zhejiang A\&F University, 311300 Hangzhou, China

Full list of author information is available at the end of the article

These authors contributed equally: Jian Zhang, Huwei Yuan, Yujuan Li
}

triploidy, tetraploidy, pentaploidy, hexaploidy, and even dodecaploidy $(2 n=12 \mathrm{x}=228)$, is abundant among willow species in nature ${ }^{8,9}$. Most shrub willow tree species are diploid, while most arbor species are allopolyploid ${ }^{10-12}$. To provide the necessary genetic characteristics of willow for scientific study, the genomes of several diploid shrub willow species, including Salix suchowensis ${ }^{13}$ and Salix purpurea (available at http://phytozome.jgi.doe.gov), were sequenced successively.

Salix matsudana Koidz is an allotetraploid arbor tree species in Salix with high stress resistance and strong adaptability. S. matsudana has been cultivated widely in China, and has thus been given the name "Chinese willow". S. matsudana has been introduced to many areas around the world, including Australia, Europe, and North America ${ }^{14}$. Selection and breeding of new stress-resistant varieties of $S$. matsudana have been conducted at several institutions, and a series of elite varieties, including "9901" and "Hailiu 1", were selected and applied in coastal regions of China. To better understand the molecular characteristics of $S$. matsudana, the first draft genome of $S$. matsudana was assembled, and the phylogenetic

\section{(c) The Author(s) 2020}

(c) (i) Open Access This article is licensed under a Creative Commons Attribution 4.0 International License, which permits use, sharing, adaptation, distribution and reproduction cc) in any medium or format, as long as you give appropriate credit to the original author(s) and the source, provide a link to the Creative Commons license, and indicate if changes were made. The images or other third party material in this article are included in the article's Creative Commons license, unless indicated otherwise in a credit line to the material. If material is not included in the article's Creative Commons license and your intended use is not permitted by statutory regulation or exceeds the permitted use, you will need to obtain permission directly from the copyright holder. To view a copy of this license, visit http://creativecommons.org/licenses/by/4.0/. 
characteristics of $S$. matsudana were revealed by comparing its genome with available genomes of $S$. suchowensis, S. purpurea, Populus trichocarpa ${ }^{15}$, and Populus euphratica ${ }^{16}$ in the same family.

\section{Results}

\section{Genome assembly and quality assessment}

According to the kmer distribution, the heterozygosity rate of S. matsudana was estimated to be $0.71 \%$ (Supplementary Fig. S1), demonstrating the highly heterozygous genome of this species. In total, $78.50 \mathrm{G}$ of raw data with a depth of $125 \times$ and Q30 of $>93.11 \%$ was obtained by the second-generation Illumina sequencing platform (Supplementary Table S1); $37.58 \mathrm{G}$ of raw data with a total base content of $35.64 \mathrm{~Gb}$, a mean length of $10.31 \mathrm{~Kb}$, and a mean read quality of 0.834 were obtained by the third-generation PacBio sequencing platform (Supplementary Table S2), and 289 G of optical data were obtained based on standard protocols. The raw data from the third-generation PacBio sequencing platform were initially assembled using Canu ${ }^{17}$ and Quickmerge ${ }^{18}$ software, followed by error correction with Pilon ${ }^{19}$ software using the second-generation sequencing data, and final scaffold extension with Irys-scaffolding ${ }^{20}$ software using the optical data. Finally, a genome of $S$. matsudana with a total size of $655.72 \mathrm{Mb}$ was obtained, for which the number of scaffolds, scaffold N50, contig N50, and GC content were $2120, \sim 12.35 \mathrm{Mb}, \sim 945.75 \mathrm{~Kb}$, and $33.55 \%$, respectively (Table 1).

After quality control of the assembled sequences, 450 core genes accounting for 98.25 of the total (458; Supplementary Table S3) and 1313 complete core genes accounting for 94.38 of the total (1440; Supplementary Table S4) were identified in the CEMGA (Core Eukaryotic Genes Mapping Approach) database and BUSCO database, respectively, demonstrating the acceptable consecutiveness, coverage rate, and accuracy of the $S$. matsudana genome constructed in this study.

\section{Genome annotation}

\section{Analysis of repeated sequences}

A repeated sequence database of the $S$. matsudana genome was constructed based on the principles of structure prediction and de novo sequencing. The genome of S. matsudana was clustered and ranked according to a previously published genetic map ${ }^{21}$. In total, $531.43 \mathrm{Mb}$ of the genome sequences (accounting for $81.43 \%$ ) was mapped onto the 38 chromosomes of $S$. matsudana, the length of which ranged from $8.80-28.42 \mathrm{Mb}$. In total, $300.41 \mathrm{Mb}$ of repeated sequences, accounting for $45.97 \%$ of the whole genome, was identified, among which the numbers of SSRs and unknown repeated sequences were 6185 and 116,059, accounting for $0.51 \%$ and $6.76 \%$ of the total genome, respectively (Supplementary Table S5).

\section{Gene prediction}

A total of 57,841 genes with a total length of $216,437 \mathrm{~Kb}$, a mean exon length of $229 \mathrm{bp}$, a mean intron length of $350 \mathrm{bp}$, and a density of 108.9 genes per $\mathrm{Mb}$ were predicted. In addition, 220 miRNAs (belonging to 27 gene families), 321 rRNAs (belonging to 4 gene families), 1557 tRNAs (belonging to 23 gene families), and 3494 pseudogenes were discovered (Fig. 1). The results from blast alignment showed that $99.49 \%$ of the genes were annotated in the NR, COG, and KEGG databases (Supplementary Table S6), while only 295 genes were not annotated in these databases.

\section{Comparisons between genomes of willow and poplar}

Comparisons of genome sequences between S. matsudana and four other species in Salicaceae, namely, $P$. trichocarpa, P. euphratica, S. suchowensis, and S. purpurea, showed that the total number of genes in $S$. matsudana were 1.53 times and 2.17 times that in S. purpurea and S. suchowensis, respectively (Table 2).

A total of 50,381 of the 57,841 genes in the $S$. matsudana genome could be clustered into 18,233 gene families, among which 13,787 were common among the five species of Salicaceae, while 302 were specific to S. matsudana (Supplementary Table S7 and Fig. 2). A total of 793 genes were included in the gene families specific to S. matsudana, and their functions were related to metabolic process, cellular process, response to stimulus, and biological regulation (Supplementary Fig. S2).

Table 1 Summary of the S. matsudana genome

\begin{tabular}{llllll}
\hline Scaffold number & Scaffold length (bp) & Scaffold N50 (bp) & Scaffold N90 (bp) & Scaffold max (bp) & Gap total length (bp) \\
\hline 2120 & $655,719,782$ & $12,349,754$ & 180,627 & $28,420,164$ & 726 \\
Contig number & Contig length (bp) & Contig N50 (bp) & Contig N90 (bp) & Contig max (bp) & GC content (\%) \\
2819 & $653,522,500$ & 945,757 & 148,606 & $5,826,838$ & 33.55 \\
\hline
\end{tabular}

Note: contig number: the number of contigs $>1 \mathrm{~Kb}$ in length; contig length (bp): the length of contigs $>1 \mathrm{~Kb}$ in length; contig N50 (bp): the length of contigs with an $\mathrm{N} 50>1 \mathrm{~Kb}$; contig $\mathrm{N} 90$ (bp): the length of contigs with an $\mathrm{N} 90>1 \mathrm{~Kb}$; contig max (bp): the length of the longest contig >1 Kb in length; gap total length (bp): total length of the gap 


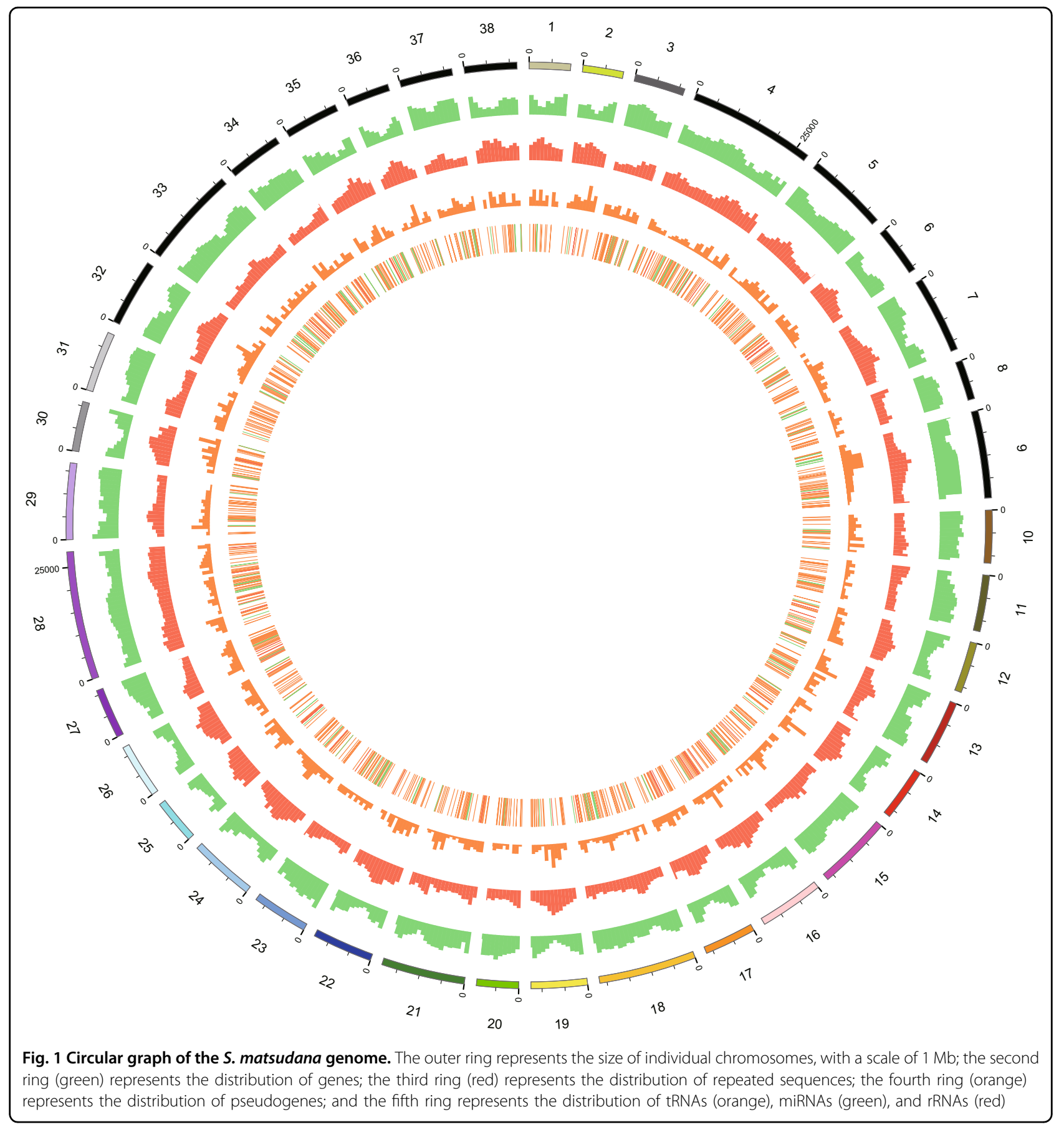

\section{Phylogenetic analysis of S. matsudana}

\section{Expansion analysis of genes in S. matsudana}

Further investigation of the expansion and contraction of gene families in the five tree species in Salicaceae demonstrated that the numbers of gene families and genes that were expanded in S. matsudana were 3128 and 20,699, respectively (Supplementary Fig. S3). The functions of these genes were mainly related to metabolic process, cellular process, cell part, cell binding, catalytic activity, and so on. In addition, a series of root development-related gene families, such as root cap, root hair defective 3 GTPbinding protein (RHD3), and transcription factor regulating root and shoot growth via Pin3, were identified as expanded families, and their gene numbers were expanded to 18,27 and 29 , respectively. 
Table 2 Comparisons between the genomes of five tree species in Salicaceae

\begin{tabular}{|c|c|c|c|c|c|}
\hline & $\begin{array}{l}\text { S. purpurea } \\
\text { (diploid) }\end{array}$ & $\begin{array}{l}\text { S. suchowensis } \\
\text { (diploid) }\end{array}$ & $\begin{array}{l}\text { S. matsudana } \\
\text { (tetraploid) }\end{array}$ & $\begin{array}{l}\text { P. euphratica } \\
\text { (diploid) }\end{array}$ & $\begin{array}{l}\text { P. trichocarpa } \\
\text { (diploid) }\end{array}$ \\
\hline Genome size & $392 \mathrm{Mb}$ & $425 \mathrm{Mb}$ & $656 \mathrm{Mb}$ & $497 \mathrm{Mb}$ & $485 \mathrm{Mb}$ \\
\hline $\begin{array}{l}\text { Assembled genome } \\
\text { sequence size }\end{array}$ & $348 \mathrm{Mb}$ & $303 \mathrm{Mb}$ & $531 \mathrm{Mb}$ & $323 \mathrm{Mb}$ & $388 \mathrm{Mb}$ \\
\hline Scaffold N50 & $190.9 \mathrm{~Kb}$ & $925.0 \mathrm{~Kb}$ & $12,349.8 \mathrm{~Kb}$ & $482.1 \mathrm{~Kb}$ & $19,500 \mathrm{~Kb}$ \\
\hline Contig N50 & $45.6 \mathrm{~Kb}$ & $17.4 \mathrm{~Kb}$ & $945.8 \mathrm{~Kb}$ & $40.4 \mathrm{~Kb}$ & $552.8 \mathrm{~Kb}$ \\
\hline Number of predicted genes & 37,865 & 26,599 & 57,841 & 34,279 & 42,950 \\
\hline Gene density (genes per Mb) & 109 & 88 & 109 & 106 & 111 \\
\hline
\end{tabular}

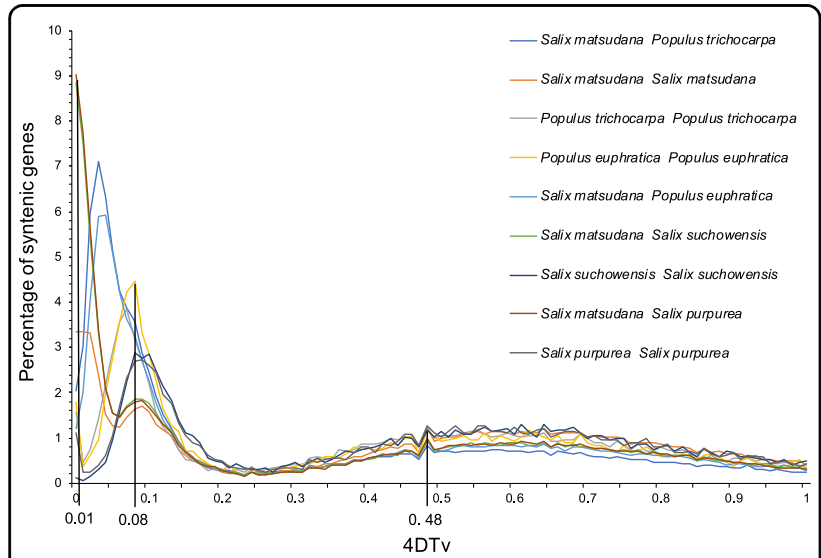

Fig. 3 The 4DTv distribution among the five tree species in Salicaceae. The $x$-coordinates and $y$-coordinates represent 4DTV distance and percentage of syntenic genes, respectively

\section{Fourfold degenerate site (4DTV) analysis of the five tree species in Salicaceae}

The duplication time of the genome was estimated by comparing the 4DTv mutation rates among genes in the collinear fragments of the five tree species in Salicaceae (Fig. 3). A genome-wide replication event was predicted to have occurred 220-358 million years ago (MYA) among the ancestors of Salicaceae, since the first peak was at 0.48 and the synonymous mutation rates $(R$ values) of Salicaceae ranged from $1.09 \times 10^{-9}$ to $0.67 \times$ $10^{-9} /$ site/year ${ }^{13}$. Similarly, a second genome-wide replication event was predicted to have occurred 36.7-59.7 MYA among species in Salicaceae, since the second peak was at 0.08 , at which time differentiation between poplar and willow was estimated to have appeared. In addition, the allotetraploid was estimated to have formed 4.6-7.5 MYA in S. matsudana, since a peak appeared at 0.01 for this species.
Long terminal repeat sequence analysis among the five tree species in Salicaceae

Among the long terminal repeat sequence (LTR) transposons in S. matsudana, 213,911, with a total length of $93,513,152 \mathrm{bp}$ and accounting for $14.31 \%$ of the repeated sequences, were Copia transposons, while 227,569, with a total length of 100,397,354 bp and accounting for $15.36 \%$ of the repeated sequences, were Gypsy transposons.

After estimation of the two types of LTRs by sequence bifurcation analysis, it was found that the insertion of LTR transposons into Salicaceae genomes began 6 MYA, while the insertion of LTR transposons began 4 MYA in the S. matsudana genome (Fig. 4), further confirming that the formation of allotetraploid S. matsudana occurred recently.

\section{Expression analysis of root-related genes in arbor and shrub willows}

Arbor and shrub willows are diverse in terms of tiller number, plant height, and primary root development. For instance, the primary roots are well developed in arbor willow, while the primary roots are not obvious, but the lateral roots are well developed in shrub willow. A series of genes were identified as rapidly evolving genes and annotated as root-related genes after comparing the two shrub willow genomes with that of $S$. matsudana. The expression levels of these genes were determined in ten arbor and shrub willow varieties, using $\operatorname{SmACTIN1}$ as a reference gene. The results showed that TCTP1 and CYP71 were differentially expressed in the roots of arbor and shrub willows (Fig. 5). Results from Arabidopsis showed that TCTP was expressed throughout the plant, but with the highest levels in meristematic regions of the shoot and root, demonstrating its functions in lateral root development, root development, and root hair cell tip growth. CYP71 is a WD40 domain cyclophilin that functions in gene repression, organogenesis, and meristem development. Based on the above results, TCTP1 


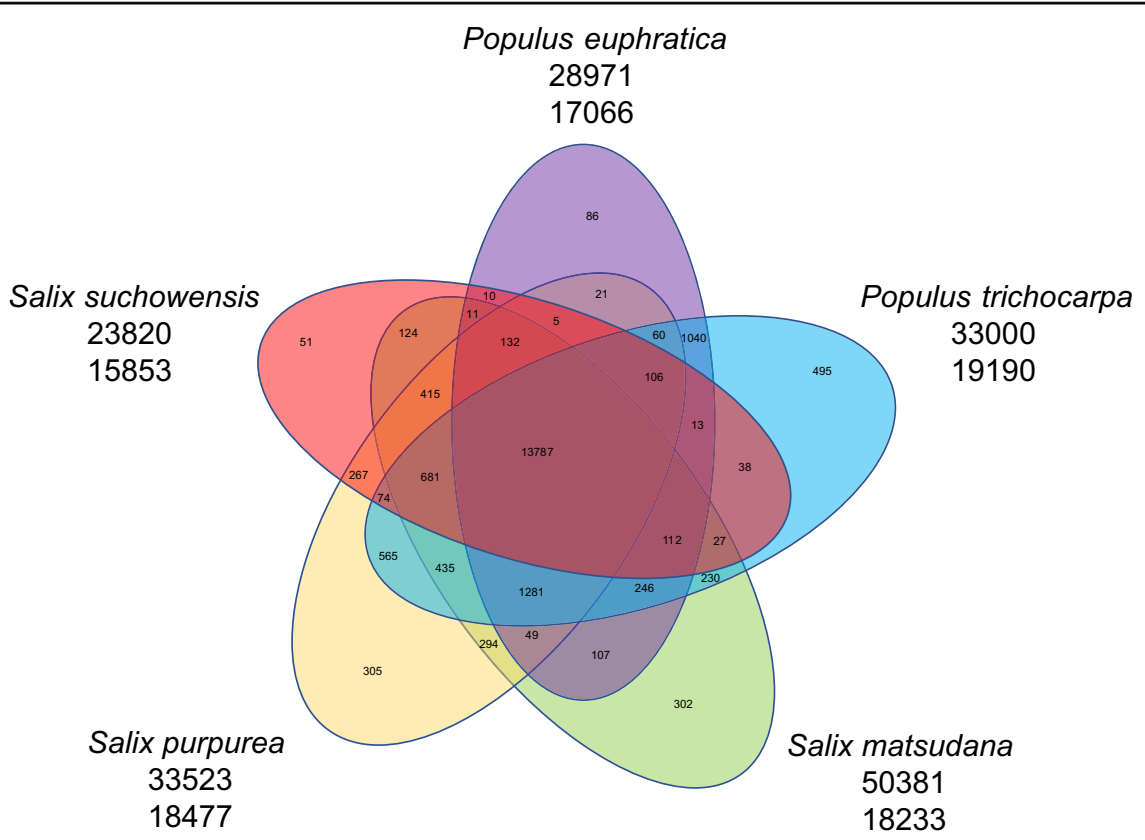

Fig. 2 The common and specific gene families in the genomes of five tree species in Salicaceae. The numbers under the five tree species represent cluster gene number and total gene family number, respecitively

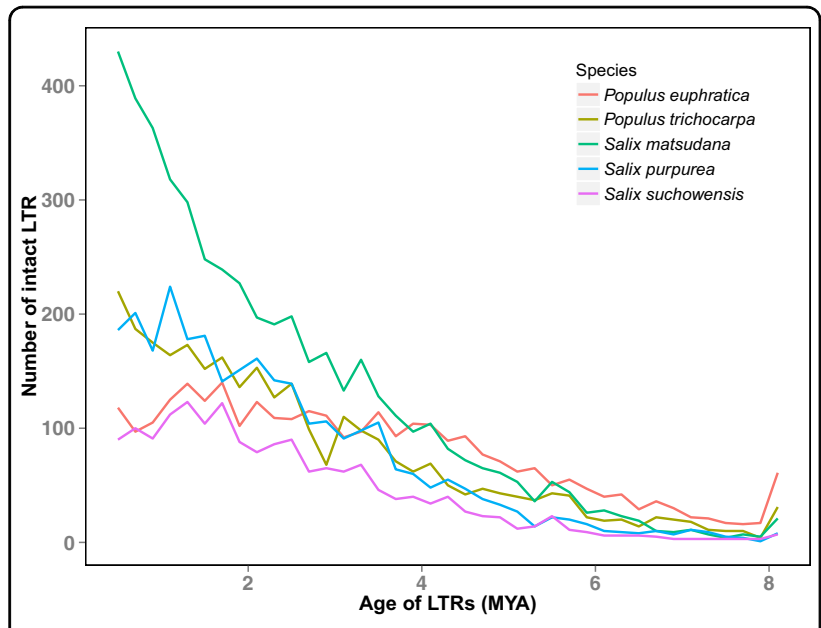

Fig. 4 Insertion time estimation for LTR transposons in the five tree species in Salicaceae. The $x$-coordinates and $y$-coordinates represent the age of LTRs and number of intact LTR, respectively

and CYP71 were predicted to be related to the differentiation of arbor and shrub willows.

\section{Analysis of chromosome differentiation and genome comparison in S. matsudana}

\section{Chromosome differentiation in the S. matsudana genome}

The chromosomes of S. matsudana are displayed in a boxplot according to identity value. Among the 38 chromosomes of $S$. matsudana, the chromosome group homologous to that of $P$. trichocarpa was identified and

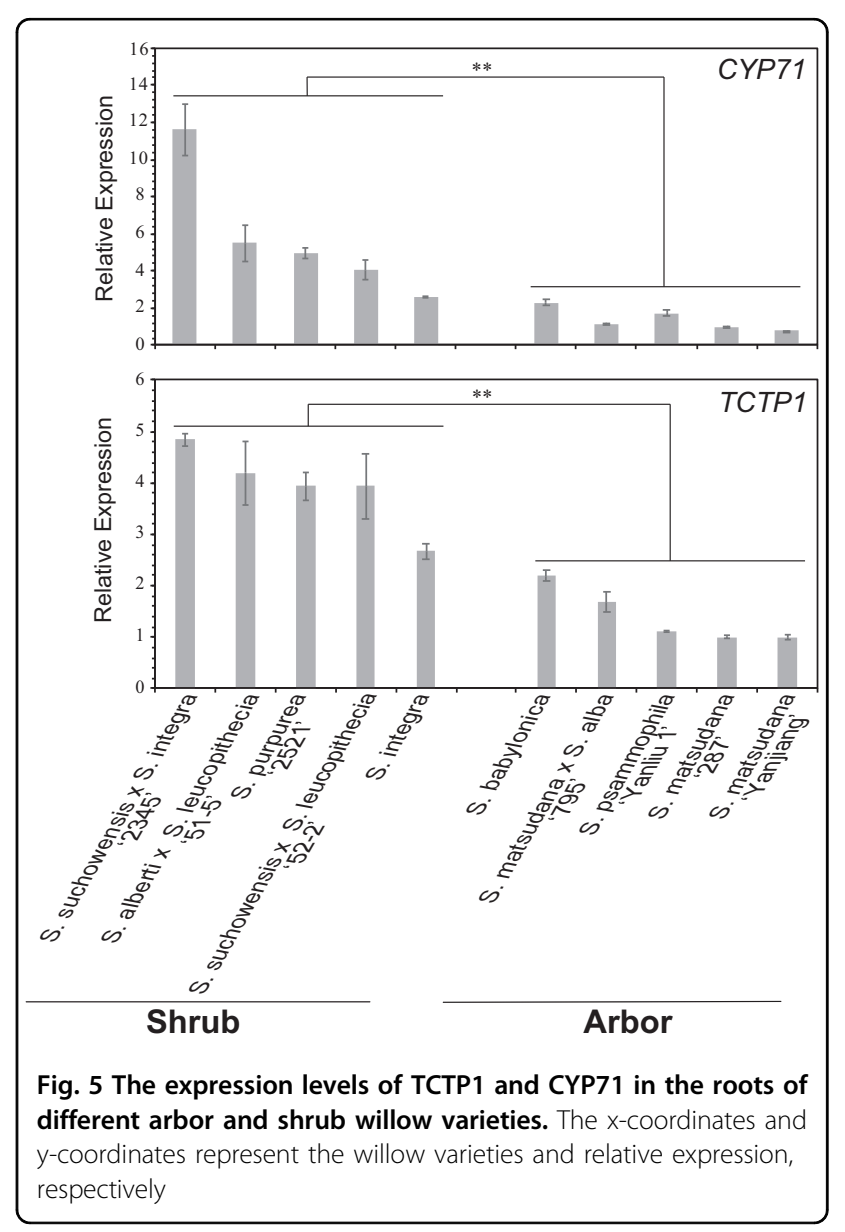




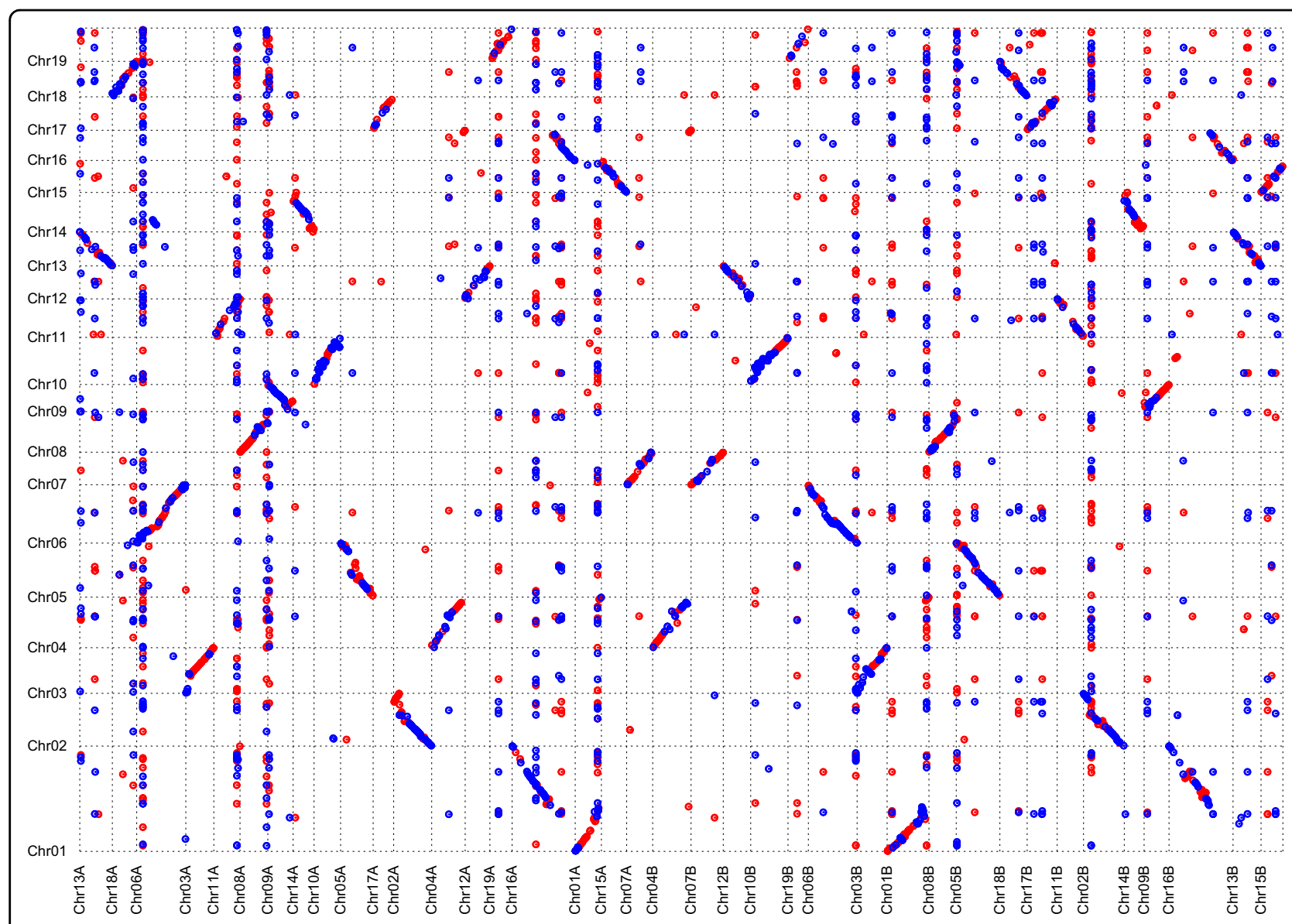

Fig. 6 The collinearity between the genomes of S. matsudana and P. trichocarpa. The $x$-coordinates and $y$-coordinates represent the genomes of S. matsudana and P. trichocarpa, respectively. Red represents positive alignment, while blue represents reverse alignment

named the A genome, while the remaining 19 chromosomes were classified as the B genome (Supplementary Fig. S4). The A genome was $252.68 \mathrm{Mb}$ in length, with a density of 95 genes per $\mathrm{Mb}$, while the $\mathrm{B}$ genome was $278.76 \mathrm{Mb}$ in length, with a density of 90 genes per $\mathrm{Mb}$. The densities of both the A and B genomes of S. matsudana were lower than those in P. trichocarpa.

The collinearity between the genomes of $S$. matsudana and $P$. trichocarpa was analyzed by MUMMER software. The results showed that the recombination events occurred between Chr1 in P. trichocarpa, and Chr1A and Chr16A in the A genome of $S$. matsudana, and similar events were also observed for the B genome of S. matsudana (Fig. 6), demonstrating that Chr1 in P. trichocarpa underwent a breakage event. One of the fragments was reconnected with Chr16 and formed Chr1 in S. matsudana, and the other fragment formed Chr1 in S. matsudana, which was consistent with the findings of previous studies ${ }^{22,23}$.

\section{Comparisons between different genome groups of S. matsudana}

Comparisons between different genome groups of S. matsudana showed that the number of genes in the A and B genomes were 23,985 and 25,107 , respectively. Collinearity analysis between the $\mathrm{A}$ and $\mathrm{B}$ genomes revealed different degrees of rearrangement between chromosomes in the $\mathrm{A}$ and $\mathrm{B}$ genomes, such as between Chr1A and Chr3B, and between Chr1B and Chr3A. In addition, duplications of some chromosome fragments were also observed. For example, Chr8A in the A genome, corresponding to $\mathrm{Chr} 8 \mathrm{~B}$ in the $\mathrm{B}$ genome, was duplicated into Chr10B (Fig. 7).

PAVs between the A and B genomes of S. matsudana were initially identified by ppsPCP ${ }^{24}$ software, further verified by searching for PAV sequences in the reference genome, and finally confirmed by comparing the PAVs with queries. The results indicated that the number of PAV mutation-related genes in the A genome compared with the B genome was 19,841 , while the number of PAV mutation-related genes in the $\mathrm{B}$ genome compared with the A genome was 12,246. GO enrichment analysis of the genes with PAVs between the A and B genomes suggested that the functions of these genes were consistent in rank between the $A$ and $B$ genomes in terms of biological process, cellular component, and molecular function (Supplementary Table S8 and Supplementary Fig. S5). For 


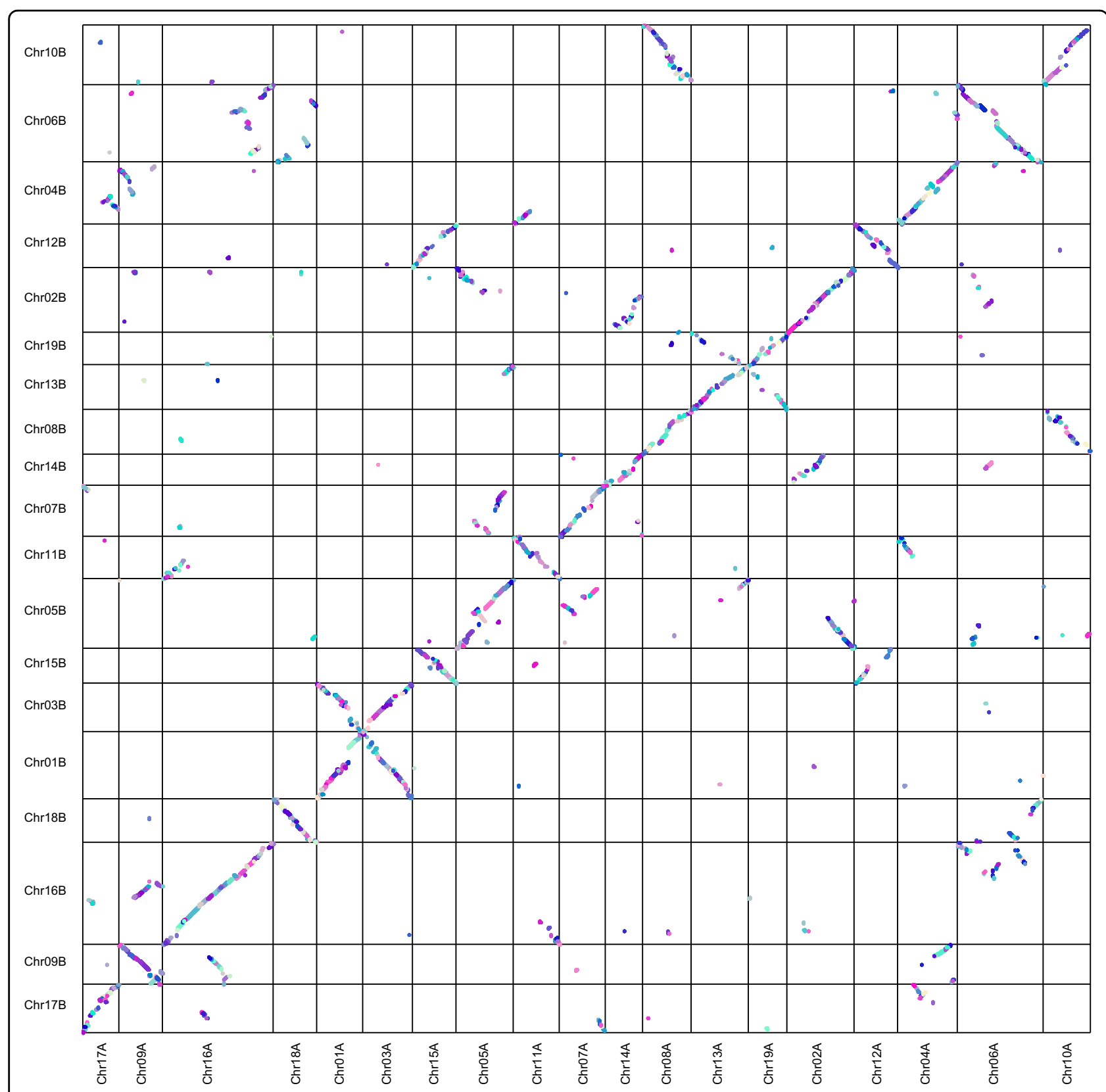

Fig. 7 The collinearity relationships between the A and B genomes of S. matsudana. The x-coordinates and $y$-coordinates represent the A and B genomes of S. matsudana, respectively

example, most of the genes with PAVs, i.e., 3693 in the A genome and 4231 in the B genome, were annotated as being associated with the metabolic process function in the biological process category, accounting for $54.28 \%$ and $55.66 \%$ of the total genes in the corresponding A and B genomes, respectively.

KEGG enrichment analysis of the genes with PAVs between the $A$ and $B$ genomes suggested that nine of the top ten significantly enriched pathways were different between the $\mathrm{A}$ and $\mathrm{B}$ genomes. The most enriched pathways were the pyruvate metabolic pathway, and the starch and sucrose metabolic pathway in the A and B genomes, with 40 and 100 genes annotated, respectively (Supplementary Fig. S6). Significant differences between the A and B genomes suggested their varying roles in regulating basal metabolism.

\section{Genome differentiation of willow}

A phylogenetic tree was constructed according to the single-copy protein sequences in the five tree species in 
Salicaceae and the differentiation time of Salicaceae ${ }^{13}$. The differentiation time of the two shrub willows was 13.3 MYA, while the differentiation of the original species conferring the A and B genomes to S. matsudana started $~ 25.2$ MYA (Supplementary Fig. S7).

\section{Discussion}

\section{Ploidy analysis of willow chromosomes}

Willows, the generic term for landscaping and energy tree species in Salicaceae, are variable in terms of chromosome ploidy, from $2 n=38$ to $12 n=228$ (refs. ${ }^{8,9}$ ). Most shrub willows are diploid, with a genome size of $\sim 400 \mathrm{Mb}$. For instance, the genome sizes of Salix triandra L. (ref. ${ }^{25}$ ) and S. suchowensis Cheng ${ }^{13,26}$. are 386 and $425 \mathrm{Mb}$, respectively. Arbor willows are mainly allopolyploid $^{10-12}$, with a genome size of $>650 \mathrm{Mb}$. For example, the genome sizes of Salix babylonica L. (ref. ${ }^{27}$ ) and Salix alba L. (ref. ${ }^{25}$ ) are 748 and $807 \mathrm{Mb}$, respectively. $S$. matsudana is a tetraploid species ${ }^{28}$. A previous study by our group estimated that the genome size of $S$. matsudana was $\sim 670 \mathrm{Mb}$ by flow cytometry ${ }^{29}$. In this study, the genome size of this species was $656 \mathrm{Mb}$ (Table 1), which was in accordance with the size in the previous study.

Chromosome doubling of somatic cells and unreduced germ cells are the main pathways for the formation of polyploids in plants during evolution ${ }^{30}$. Autopolyploids are polyploids derived from the same species, while allopolyploids are polyploids originating from different species $^{31}$. For example, hexaploid bread wheat (Triticum aestivum L., AABBDD) originated initially from a hybridization event between Triticum urartu (AA) and T. aestivum $\mathrm{L}$. (BB) to form the tetraploid Triticum turgidum (AABB), followed by the hybridization event between Aegilops tauschii (DD) and T. turgidum ${ }^{32}$. The origin of tetraploid Gossypium hirsutum L. (AADD) and Gossypium barbadense L. (AADD) was dated back to 1.5 MYA, which involved the formation of the A genome donated by Gossypium herbaceum L. or Gossypium arboreum L., and the hybridization event between the A genome and D genome (donated by Gossypium raimondii Ulbrich) ${ }^{33}$. The genome comparison in the present study suggested that 19 of the 38 total chromosomes in S. matsudana were consistent with those in the $P$. trichocarpa genome (Fig. 6), indicating that S. matsudana might have originated from hybridization between the ancestors of $P$. trichocarpa and another species. 4DTv combined transposon analysis predicted that the genome of $S$. matsudana doubled 4 MYA (Figs. 3 and 4), indicating that the formation of the allotetraploid in this species might have occurred during this period.

\section{Split between arbor and shrub willows}

Arbors and shrubs are two growth forms specific to tree species. Different types of arbors and shrubs have been observed simultaneously in some willow species ${ }^{34}$. In this study, the split between the two shrub willow species $S$. suchowensis and $S$. purpurea was estimated to have occurred 13.3 MYA, while the split between the species donating the A genome in S. matsudana and shrub willow occurred 19.0 MYA (Supplementary Fig. S6). However, because of the limited number of willow genomes sequenced, the exact split time between arbor and shrub willows needs to be further studied.

Arbor and shrub willows displayed significant phenotypic variations in terms of root, branch, and crown growth. The differences in root growth between arbor and shrub willows have been rarely studied, using molecular biology methods. In the current study, TCTP1 and CYP71, screened from rapidly evolving genes of the two shrub willows in comparison to $S$. matsudana, were identified as differentially expressed genes between the roots of arbor and shrub willow varieties. It was reported that TCTP1 functioned in lateral root development and determined total root length in Arabidopsis ${ }^{35}$. In addition, TCTP induces the formation of adventitious roots in tobacco $^{36}$. CYP71 functions in gene repression and organogenesis, and abnormalities in CYP71 result in the ectopic activation of homologous genes regulating meristem development. Mutants of CYP71 displayed phenotypes of low apical meristem activity and disrupted root growth $^{37}$. TCTP1 and CYP71 were highly expressed in the shrub willow varieties, but weakly expressed in the arbor willow varieties (Fig. 5), suggesting that they might have been involved in the split between arbor and shrub willows. Further functional analysis of these genes would provide insight into the nature of the split between arbor and shrub willows.

\section{Differentiation time between the A and B genomes of $S$. matsudana}

Most species have undergone $>2$ whole-genome duplications (WGDs), which facilitated their survival and adaptation to different environmental conditions ${ }^{38}$. The results from this study indicated that species in Salicaceae had undergone at least two WGD events, and the second WGD occurred 36.7-59.7 MYA (Fig. 3).

Fossil records and genome sequences are commonly used to estimate the time of species differentiation. Poplar and willow belong to the same family but different genera. Previous studies based on fossil records revealed that the differentiation of Salicaceae occurred $\sim 45-48 \mathrm{MYA}^{39-41}$. The differentiation time of Salicaceae was estimated to be 12 (ref. ${ }^{42}$ ), 48 (ref. ${ }^{41}$ ), and $52 \mathrm{MYA}^{13}$ based on sequencing results for the chloroplast, plastid, and nuclear genomes of S. suchowensis, respectively. The variation in differentiation time might be caused by differences in sample selection, which could lead to variation in the correction coefficient and even the final result. The 
differentiation of the A and B genomes of S. matsudana (Supplementary Fig. S7) was deduced to be 25.2 MYA based on the split time of Salicaceae estimated by the $S$. suchowensis genome, as well as the single-copy orthologs within the whole genome.

\section{Methods}

\section{Genome sequencing}

Fresh leaves of S. matsudana were collected in Changjiang town, Rugao city, Jiangsu Province, China, frozen in liquid nitrogen immediately, and stored at $-80^{\circ} \mathrm{C}$ for subsequent genomic DNA extraction and library construction. The genomic DNA was fragmented by g-TUBE, repaired, ligated with a dumbbell-shaped adaptor, and digested with exonuclease. The target fragments were screened by BluePippin and used for library construction. Second-generation sequencing was performed according to the protocols provided by the Illumina company. Assembly of the genome sequences from the thirdgeneration sequencing was conducted by the PacBio technique. All sequencing procedures were performed by the BioMarker Technologies Company (Beijing, China).

\section{Genome annotation}

\section{Annotation of repeated sequences}

The repeated sequence database of the S. matsudana genome was constructed initially based on the structure prediction and de novo prediction, using LTR FINDER ${ }^{43}$, MITE-Hunter ${ }^{44}$, RepeatScout ${ }^{45}$, and PILER-DF ${ }^{46}$ software. The database was classified with PASTEClassifier ${ }^{47}$ and combined with the Repbase ${ }^{48}$ database to form the final repeated sequence database. The repeated sequences were annotated based on the final repeated sequence database using RepeatMasker ${ }^{49}$ software.

\section{Gene expression}

Three strategies, namely, de novo prediction, homologous species-based prediction, and unigene-based prediction, were adopted for gene prediction. The prediction results from different strategies were integrated by $\mathrm{EVM}^{50}$ software. GENSCAN ${ }^{51}$, Augustus ${ }^{52}$, GlimmerHMM ${ }^{53}$, GeneID $^{54}$, and SNAP ${ }^{55}$ were used for de novo prediction. $P$. trichocarpa and $P$. euphratica were selected for homologous species-based prediction using GeMoMa ${ }^{56}$. PASA $^{57}$ was used for unigene-based prediction.

\section{Annotation of gene functions}

The predicted gene sequences were subjected to BLAST $^{58}$ alignment with the $\mathrm{NR}^{59}, \mathrm{COG}^{60}$, and KEGG ${ }^{61}$ functional databases. Functional annotations in the $\mathrm{GO}^{62}$ database were compared with the NR database alignment result using Blast $2 \mathrm{GO}^{63}$. In addition, the predicted genes were also subjected to COG, KEGG, and GO enrichment analyses.

\section{Comparative genomic analysis}

The protein sequences of $S$. matsudana were aligned with those in related species, including $S$. suchowensis, $S$. purpurea, $P$. trichocarpa, and $P$. euphratica, to analyze gene duplication within species, gene evolution between species and the classification of species-specific genes. Clustering analysis of gene families was conducted by OrthoMCL ${ }^{64}$ software. The $4 \mathrm{DT}$ v mutation rate was calculated for each of the homologous gene pairs between and within species. LTR sequences with scores $\geq 6$ were detected in the genome by LTR FINDER ${ }^{43}$ and PS SCAN $^{65}$, but the repeated sequences detected by LTR FINDER were filtered. The flanking sequences of LTRs were extracted and aligned with MUSCLE ${ }^{66}$. The degrees of sequence differences were calculated by DistMat software, with the Kimura model and a $7.3 \times 10^{-9}$ molecular clock.

\section{qRT-PCR of CYP71 and TCTP1}

The branches of " 2345 " (S. suchowensis $\times S$. integra), "51-5" (S. alberti $\times$ S. leucopithecia), "2521" (S. purpurea), "52-2" (S. suchowensis $\times$ S. leucopithecia), S. integra, S. babylonica, "Yanliu 1" (S. psammophila), "795" (S. matsudana $\times$ S. alba), "287" and "Yanjiang" (S. matsudana) were collected and clipped to a length of $10 \mathrm{~cm}$ and diameter of $1 \mathrm{~cm}$. Three biological replicates with ten cuttings per replicate were analyzed for each variety. After 15 days of hydroponic culture, the roots of each cutting were collected and stored at $-80^{\circ} \mathrm{C}$. Total RNA from the roots of the ten varieties was extracted using an RNAprep Pure Plant kit (Tiangen). The expression levels of the two genes (CYP71 and TCTP1) in each biological replicate were measured by qRT-PCR using three technological replicates. A PrimeScript ${ }^{\mathrm{TM}}$ RT Reagent Kit (Takara) was used for reverse transcription of the RNAs. Reverse transcription was performed at $37^{\circ} \mathrm{C}$ for $30 \mathrm{~min}$ and $85^{\circ} \mathrm{C}$ for 5 s. A One-Step SYBR Primer Script PLUS RT-PCR Kit (Takara) was used for qRT-PCR of the CYP71 and TCTP1 genes. The reaction was performed at $95^{\circ} \mathrm{C}$ for $2 \mathrm{~min}$, followed by 40 cycles of $95^{\circ} \mathrm{C}$ for $5 \mathrm{~s}, 56^{\circ} \mathrm{C}$ for $30 \mathrm{~s}$, and $72{ }^{\circ} \mathrm{C}$ for $20 \mathrm{~s}$. The Actin gene in Salix was used as the reference gene. The qRT-PCR primers are listed in Supplementary Table S9. Relative expression was measured using the $2^{-\Delta \Delta C t}$ method. The expression level of " 287 " ( $S$. matsudana) was selected as the reference level (with a relative expression of 1.0).

\section{Construction of the phylogenetic tree}

The A and B genomes of S. matsudana, and the genomes of S. suchowensis, S. purpurea, P. trichocarpa, and P. euphratica were used for phylogenetic analysis. The sequence of each single-copy gene family was aligned with MAFFT (v7.205), while the regions with significant differences were removed by Gblocks (v0.91b). Finally, 
supergenes were obtained by connecting all the aligned gene families in each species. ModelFinder in IQ-TREE was used for model testing, and the best model was predicted to be JTT $+\mathrm{F}+\mathrm{I}+\mathrm{G} 4$. The maximum likelihood method was used to construct a rooted phylogenetic tree. $P$. trichocarpa was selected as the outgroup species, and the number of bootstraps was set to 1000 . Split times and the gradient and Hessian parameters were calculated by MCMCTREE in PAML (v4.9i) software. Visualization of the phylogenetic tree with split times was performed by MCMCTreeR.

\section{Collinearity analysis between the A and B genomes of $S$. matsudana}

The similar gene pairs, with the criteria of an $E$ value < $1 \mathrm{e}-5$ and a $C$ score $>0.5$ ( $C$ scores were filtered by JCVI software), were identified by comparing gene sequences between species using Diamond (v0.9.29.130). gff3 was used to determine whether similar gene pairs were adjacent in the genome.

\section{Acknowledgements}

This work was supported by the National Natural Science Foundation of China (31971681), Jiangsu Province Forestry Science and Technology Innovation and Promotion Project (LYKJ [2018]36) and Nantong University Scientific Research Start-up Project for Introducing Talents (18R08).

\section{Author details}

'Key Lab of Landscape Plant Genetics and Breeding, School of Life Science, Nantong University, 226019 Nantong, China. ${ }^{2}$ State Key Laboratory of Subtropical Silviculture, Zhejiang A\&F University, 311300 Hangzhou, China. ${ }^{3}$ Jiangsu Riverine Institute of Agricultural Sciences, 226541 Nantong, Jiangsu, China. ${ }^{4}$ College of Biological Sciences and Technology, Beijing Forestry University, 100083 Beijing, China. ${ }^{5}$ National Engineering Laboratory of Tree Breeding, Beijing Forestry University, 100083 Beijing, China

\section{Author contributions}

J.Z. and J.C.X. conceived and designed the project. Y.J.L. and Y.N.J. performed the experiments. Y.H.C. and G.Y.L. conducted the gene expression analysis. M.X. Y., C.M.Y., B.L.L., and F.Z. analyzed the data. H.W.Y. wrote the paper. All authors approved the final version of the manuscript.

\section{Conflict of interest}

The authors declare that they have no conflict of interest.

Supplementary Information accompanies this paper at (https://doi.org/ 10.1038/s41438-020-00424-8).

Received: 24 June 2020 Revised: 16 September 2020 Accepted: 17 September 2020

Published online: 01 December 2020

\section{References}

1. Gullberg, U. Towards making willows pilot species for coppicing production. For. Chron. 69, 721-726 (1993).

2. Lindegaard, K. N. \& Barker, J. Breeding willows for biomass. Asp. Appl Biol. 49 , 155-162 (1997).

3. Zhang, J. et al. The genetic architecture of growth traits in Salix matsudana under salt stress. Hortic. Res. 4, 17024 (2017).

4. Polt, P., Sjödin, P., Weih, M., Rönnberg-Wästljung, A. C. \& Berlin, S. Genomewide transcriptional and physiological responses to drought stress in leaves and roots of two willow genotypes. BMC Plant Biol. 15, 244 (2015).
5. Hangs, R. D., Schoenau, J. J., Van Rees, K. \& Steppuhn, H. Examining the salt tolerance of willow (Salix spp.) bioenergy species for use on salt-affected agricultural lands. Can. J. Plant Sci. 91, 509-517 (2011).

6. Yanitch, A. et al. Transcriptomic response of purple willow (Salix purpurea) to arsenic stress. Front. Plant Sci. 8, 1115 (2017).

7. Aronsson, P., Dahlin, T. \& Dimitriou, I. Treatment of landfill leachate by irrigation of willow coppice-plant response and treatment efficiency. Environ. Pollut. 158, 795-804 (2010).

8. Suda, Y. \& Argus, G. W. Chromosome numbers of some North American Salix. Brittonia 20, 191-197 (1968).

9. Macalpine, W. J., Shield, I. F., Trybush, S. O., Hayes, C. M. \& Karp, A. Overcoming barriers to crossing in willow (Salix spp.) breeding. Asp. Appl. Biol. 90, 173-180 (2008).

10. Argus, G. W. Infrageneric classification of Salix (Salicaceae) in the new world. Syst. Bot. Monogr. 52, 1-121 (1997).

11. Barcaccia, G., Meneghetti, S., Albertini, E., Triest, L. \& Lucchin, M. Linkage mapping in tetraploid willows: segregation of molecular markers and estimation of linkage phases support an allotetraploid structure for Salix albax Salix fragilis interspecific hybrids. Heredity 90, 169-180 (2003).

12. Barcaccia, G., Meneghetti, S., Lucchin, M. \& De Jong, H. Genetic segregation and genomic hybridization patterns support an allotetraploid structure and disomic inheritance for Salix species. Diversity 6, 633-651 (2014).

13. Dai, X. et al. The willow genome and divergent evolution from poplar after the common genome duplication. Cell Res. 24, 1274-1277 (2014).

14. Qiao, G. et al. Comparative proteomic analysis of responses to salt stress in Chinese willow (Salix matsudana Koidz). Plant Mol. Biol. Rep. 32, 814-827 (2014).

15. Tuskan, G. A. et al. The genome of black cottonwood, Populus trichocarpa (Torr. \& Gray). Science 313, 1596-1604 (2006).

16. $\mathrm{Ma}$, $\mathrm{T}$. et al. Genomic insights into salt adaptation in a desert poplar. Nat. Commun. 4, 1-9 (2013).

17. Koren, S. et al. Canu: scalable and accurate long-read assembly via adaptive Kmer weighting and repeat separation. Genome Res. 27, 722-736 (2017).

18. Chakraborty, M. et al. Contiguous and accurate de novo assembly of metazoan genomes with modest long read coverage. Nucleic Acids Res. $\mathbf{4 4}$ e147 (2016)

19. Chin, C. et al. Phased diploid genome assembly with single-molecule real-time sequencing. Nat. Methods 13, 1050-1054 (2016).

20. Jennifer, S. \& NicHerndon. Irys-Scaffolding: Irys-scaffolding Tools Version 1.0.0 https://doi.org/10.5281/zenodo.18591 (2015).

21. Zhang, J. et al. A high-density genetic map of tetraploid Salix matsudana using specific length amplified fragment sequencing (SLAF-seq). PLOS ONE 11, e0157777 (2016).

22. Hou, J. et al. Major chromosomal rearrangements distinguish willow and poplar after the ancestral "Salicoid" genome duplication. Genome Biol. Evol. 8, 1868-1875 (2016).

23. Hou, J., Wei, S., Pan, H., Zhuge, Q. \& Yin, T. Uneven selection pressure accelerating divergence of Populus and Salix. Hortic. Res. 6, 37 (2019).

24. Qamar, M. T. et al. ppsPCP: a plant presence/absence variants scanner and pan-genome construction pipeline. Bioinformatics 35, 4156-4158 (2019).

25. Thibault, J. Nuclear DNA amount in pure species and hybrid willows (Salix): a flow cytometric investigation. Can. J. Bot. 76, 157-165 (1998).

26. Serapiglia, M. J., Gouker, F. E. \& Smart, L. B. Early selection of novel triploid hybrids of shrub willow with improved biomass yield relative to diploids. BMC Plant Biol. 14, 74 (2014).

27. Horjales, M. Cantidades de DNA nuclear en árboles y arbustos. Nova Acta Cient. Compostel. (Bioloxía) 13, 23-33 (2003).

28. Zhang, M. L. A preliminary cladistic study on the multistaminal willows (Salix) in China. Bull. Bot. Res. 3, 299-305 (1994).

29. Zhang, J. et al. Determination of chromosome ploidy and genome size of Salix matsudana based on flow cytometry. Jiangsu Agr. Sci. 46, 40-43 (2018).

30. Ramsey, J. \& Schemske, D. W. Pathways, mechanisms, and rates of polyploid formation in flowering plants. Annu. Rev. Ecol. Syst. 29, 467-501 (1998).

31. Doyle, J. J. \& Egan, A. N. Dating the origins of polyploidy events. N. Phytol. 186, 73-85 (2010).

32. Haider, $\mathrm{N}$. The origin of the B-genome of bread wheat (Triticum aestivum $\mathrm{L}$.) Russ. J. Genet. 49, 263-274 (2013).

33. Sattler, M. C., Carvalho, C. R. \& Clarindo, W. R. The polyploidy and its key role in plant breeding. Planta 243, 281-296 (2016).

34. Tu, Z. Y. Willow Breeding And Cultivation (Jiangsu Science \& Technology Press, Nanjing, 1982). 
35. Branco, R. \& Masle, J. Systemic signalling through translationally controlled tumour protein controls lateral root formation in Arabidopsis. J. Exp. Bot. 70, 3927-3940 (2019).

36. Toscano-Morales, R., Xoconostle-Cázares, B., Martínez-Navarro, A. C. \& RuizMedrano, R. AtTCTP2 mRNA and protein movement correlates with formation of adventitious roots in tobacco. Plant Signal Behav. 11, e1071003 (2016).

37. $\mathrm{Li}, \mathrm{H}$. et al. A WD40 domain cyclophilin interacts with histone $\mathrm{H3}$ and functions in gene repression and organogenesis in Arabidopsis. Plant Cell. 19, 2403-2416 (2007)

38. Yang, H. M. GENOMICS (China Science Press, 2016).

39. Boucher, L. D., Manchester, S. R. \& Judd, W. S. An extinct genus of Salicaceae based on twigs with attached flowers, fruits, and foliage from the Eocene Green River Formation of Utah and Colorado, USA. Am. J. Bot. 90, 1389-1399 (2003).

40. Manchester, S. R., Judd, W. S. \& Handley, B. Foliage and fruits of early poplars (Salicaceae: Populus) from the Eocene of Utah, Colorado, and Wyoming. Int. J. Plant Sci. 167, 897-908 (2006).

41. Wu, J. et al. Phylogeny of Salix subgenus Salix sl (Salicaceae): delimitation, biogeography, and reticulate evolution. BMC Evol. Biol. 15, 31 (2015).

42. Hohmann, N., Wolf, E. M., Lysak, M. A. \& Koch, M. A. A time-calibrated road map of Brassicaceae species radiation and evolutionary history. Plant Cell. 27, 2770-2784 (2015).

43. Xu, Z. \& Wang, H. LTR_FINDER: an efficient tool for the prediction of full-length LTR retrotransposons. Nucleic Acids Res. 35, W265-W268 (2007).

44. Han, Y. \& Wessler, S. R. MITE-Hunter: a program for discovering miniature inverted-repeat transposable elements from genomic sequences. Nucleic Acids Res. 38, e199 (2010).

45. Price, A. L., Jones, N. C. \& Pevzner, P. A. De novo identification of repeat families in large genomes. Bioinformatics 21, i351-i358 (2005).

46. Edgar, R. C. \& Myers, E. W. PILER: identification and classification of genomic repeats. Bioinformatics 21, i152-i158 (2005).

47. Wicker, T. et al. A unified classification system for eukaryotic transposable elements. Nat. Rev. Genet. 8, 973-982 (2007).

48. Jurka, J. et al. Repbase update, a database of eukaryotic repetitive elements. Cytogenet. Genome Res. 110, 462-467 (2005).

49. Tarailo Graovac, M. \& Chen, N. Using RepeatMasker to identify repetitive elements in genomic sequences. Curr. Prot. Bioinformatics. 25, 4-10 (2009).
50. Haas, B. J. et al. Automated eukaryotic gene structure annotation using EVidenceModeler and the Program to Assemble Spliced Alignments. Genome Biol. 9, R7 (2008).

51. Burge, C. \& Karlin, S. Prediction of complete gene structures in human genomic DNA. J. Mol. Biol. 268, 78-94 (1997).

52. Stanke, M. \& Waack, S. Gene prediction with a hidden Markov model and a new intron submodel. Bioinformatics 19, i215-i225 (2003).

53. Majoros, W. H., Pertea, M. \& Salzberg, S. L. TigrScan and GlimmerHMM: two open source ab initio eukaryotic gene-finders. Bioinformatics 20, 2878-2879 (2004).

54. Blanco, E., Parra, G. \& Guigó, R. Using geneid to identify genes. Curr. Prot. Bioinformatics. 18, 3-4 (2007).

55. Korf, I. Gene finding in novel genomes. BMC Bioinformatics 5, 59 (2004).

56. Keilwagen, J. et al. Using intron position conservation for homology-based gene prediction. Nucleic Acids Res. 44, e89 (2016).

57. Campbell, M. A., Haas, B. J., Hamilton, J. P., Mount, S. M. \& Buell, C. R. Comprehensive analysis of alternative splicing in rice and comparative analyses with Arabidopsis. BMC Genomics. 7, 327 (2006).

58. Altschul, S. F., Gish, W., Miller, W., Myers, E. W. \& Lipman, D. J. Basic local alignment search tool. J. Mol. Biol. 215, 403-410 (1990).

59. Marchler-Bauer, A. et al. CDD: a Conserved Domain Database for the functional annotation of proteins. Nucleic Acids Res. 39, D225-D229 (2010).

60. Tatusov, R. L. et al. The COG database: new developments in phylogenetic classification of proteins from complete genomes. Nucleic Acids Res. 29, 22-28 (2001).

61. Kanehisa, M. \& Goto, S. KEGG: kyoto encyclopedia of genes and genomes. Nucleic Acids Res. 28, 27-30 (2000).

62. Dimmer, E. C. et al. The UniProt-GO annotation database in 2011. Nucleic Acids Res. 40, D565-D570 (2012).

63. Conesa, A. et al. Blast2GO: a universal tool for annotation, visualization and analysis in functional genomics research. Bioinformatics 21, 3674-3676 (2005).

64. Li, L., Stoeckert, C. J. \& Roos, D. S. OrthoMCL: identification of ortholog groups for eukaryotic genomes. Genome Res. 13, 2178-2189 (2003).

65. Dan, S. P. SIGNAL SCAN: a computer program that scans DNA sequences for eukaryotic transcriptional elements. Comput. Appl. Bio. 7, 203 (1991).

66. Edgar, R. C. MUSCLE: multiple sequence alignment with high accuracy and high throughput. Nucleic Acids Res. 32, 1792-1797 (2004). 